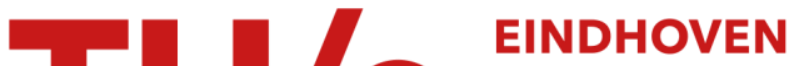 UNIVERSITY OF TECHNOLOGY
}

\section{Transforming functional database schemes to relational representations}

Citation for published version (APA):

Aerts, A. T. M., De Bra, P. M. E., \& Hee, van, K. M. (1991). Transforming functional database schemes to relational representations. (Computing science notes; Vol. 9117). Technische Universiteit Eindhoven.

Document status and date:

Published: 01/01/1991

\section{Document Version:}

Publisher's PDF, also known as Version of Record (includes final page, issue and volume numbers)

\section{Please check the document version of this publication:}

- A submitted manuscript is the version of the article upon submission and before peer-review. There can be important differences between the submitted version and the official published version of record. People interested in the research are advised to contact the author for the final version of the publication, or visit the $\mathrm{DOI}$ to the publisher's website.

- The final author version and the galley proof are versions of the publication after peer review.

- The final published version features the final layout of the paper including the volume, issue and page numbers.

Link to publication

\section{General rights}

Copyright and moral rights for the publications made accessible in the public portal are retained by the authors and/or other copyright owners and it is a condition of accessing publications that users recognise and abide by the legal requirements associated with these rights.

- Users may download and print one copy of any publication from the public portal for the purpose of private study or research.

- You may not further distribute the material or use it for any profit-making activity or commercial gain

- You may freely distribute the URL identifying the publication in the public portal.

If the publication is distributed under the terms of Article 25fa of the Dutch Copyright Act, indicated by the "Taverne" license above, please follow below link for the End User Agreement:

www.tue.nl/taverne

Take down policy

If you believe that this document breaches copyright please contact us at:

openaccess@tue.nl

providing details and we will investigate your claim. 
Eindhoven University of Technology

Department of Mathematics and Computing Science

Transforming Functional Database Schemes to Relational Representations

by

A.T.M. Aerts P.M.E. de Bra K.M. van Hee

Computing Science Note 91/17

Eindhoven, September 1991 
COMPUTING SCIENCE NOTES

This is a series of notes of the Computing Science Section of the Department of Mathematics and Computing Science Eindhoven University of Technology. Since many of these notes are preliminary versions or may be published elsewhere, they have a limited distribution only and are not for review.

Copies of these notes are available from the author.

Copies can be ordered from:

Mrs. F. van Neerven

Eindhoven University of Technology

Department of Mathematics and Computing Science

P.O. Box 513

$5600 \mathrm{MB}$ EINDHOVEN

The Netherlands

ISSN 0926-4515

All rights reserved

editors: prof.dr.M.Rem

prof.dr.K.M.van Hee. 


\title{
Transforming Functional Database Schemes to Relational Representations
}

\author{
A. T. M. Aerts ${ }^{1}$, P. M.E. De Bra ${ }^{2}$ and K. M. van $\mathrm{Hee}^{3}$ \\ Department of Mathematics and Computing Science \\ Eindhoven University of Technology \\ Eindhoven \\ The Netherlands
}

\begin{abstract}
Semantic database models have become a popular tool for designing database schemes, despite the lack of an exact and efficient way to transform these schemes into (relational) database schemes for which efficient implementations exist and are widely available. This paper presents a precise and linear-time algorithm for transforming functional database schemes into relational ones.

The functional database model has a graphical representation which is easily understood, but it also has a formal semantics based on set-theory. Hence comprehensible and mathematically exact database design can be combined using this model. By using our algorithm one can transform a functional database scheme into a relational scheme with a minimal number of relations (tables). The generated schemes are always in Fifth Normal Form (and hence also in Boyce-Codd Normal Form). Furthermore there is no need for artificially introduced primary keys to simulate the concept of "object-identity".
\end{abstract}

1 email: wsinatma@win.tue.nl

2email: debraQwin.tue.nl

${ }^{3}$ email: wsinhee@win.tue.nl 


\section{Introduction}

The Relational Database Model [1,2,3] provides a solid theoretical background for reasoning about databases, for formulating queries and updates, and for describing constraints. However, modeling a real-world situation using a relational database model is non-trivial at best. One cannot easily and intuitively define relations and constraints that form a useful and efficient representation of the real-world situation. The Functional Database Model [4] does provide such an intuitive tool, and also has a formal basis [5]. It has been proven successful in many database-design projects performed (mostly by students) for small and medium-size companies [5,6]. A graphical representation of objects, functions, and several types of constraints enable the designer to generate a visualization of the database scheme that can easily be understood by non-experts.

In order to use the Functional Database Model in real applications, an algorithm has been developed to generate a relational database scheme from a functional scheme. This is possible because in our version of the Functional Database Model a clear distinction is made between two levels: an object oriented level where the major concern is the construction of a model of the real world situation of interest, and a value oriented level, where emphasis is placed on the construction of a database model for the information system. At the first level there are just object and property types, modeling the various kinds of objects in the real world and their (functional) relationships. At the second level the representations of these types in the information system are introduced. Such a separation of concerns-modeling versus representing -is not explicitely present in Shipman's FDM where in addition also 'printable' types, such as 'STRING' and 'INTEGER', occur in the model at the same level as entitytypes.

Given a functional database model at the object oriented level, we could opt for a functional representation model, i.e. construct representations in terms of sets of object(identifier)s and functions. In this paper a relational representation scheme is chosen, i.e. a representation in terms of sets of tuples. A third alternative would be to use a network database model. Note that at this stage representations are constructed in terms of the mathematical constructs underlying each model, not in terms of the data types available in some specific database management system.

The algorithm produces a relational database scheme in Boyce-Codd Normal Form. The generated scheme does not contain multivalued dependencies or join dependencies, hence it also is in Fifth Normal Form. In this respect our approach has a clear advantage over related approaches using for instance the Entity-Relationship Model (see [7]) in which functional dependencies are not made explicit at the semantic level and have to be given extensive consideration in a separate normalization step. We supplement our basic algorithm with a few optimization steps to avoid the generation of unnecessary relations.

On several occasions the 'pure' Functional Model $[5,6]$ has been a bit too restrictive because it only provides functional relationships between objects. Therefore, like in [4], we allow the use of set-valued functions, which we replace by an additional object type and two mono-valued functions during the transformation from a functional to a relational scheme.

By using a transformation algorithm which carefully selects the relations that are needed to represent the structure of the functional database scheme we can 
employ the functional database model as a modeling tool. Classical database applications, among others, can be modeled elegantly using FDM and for such applications relational database systems have proven to provide excellent performance. When using a high-performance relational database management system, with appropriate indices, one should not suffer a large performance penalty compared to the object-oriented database management systems that exist today.

\section{The Functional Database Model}

The Functional Database Model (FDM)[6, 4] is a member of the family of Semantic Database Models (see for example [8]). A database scheme in FDM has a mathematical definition, but also a graphical representation which is easier to understand. Because the database scheme describes the structure of the part of the real world we wish to describe by means of a database, we call it a Structure Scheme. The equivalent graphical representation is called a Structure Diagram.

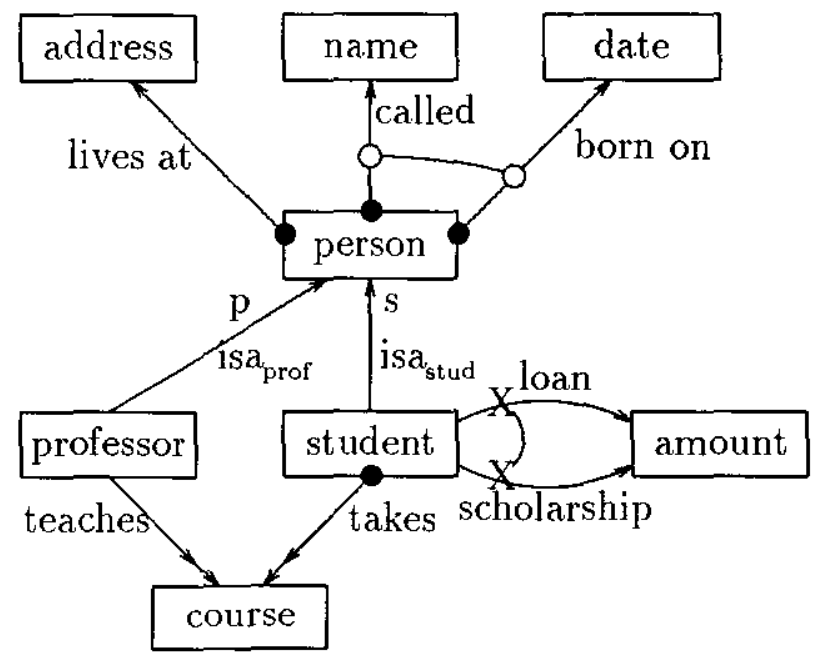

Figure 1: Structure Diagram for a University example

An example of a structure diagram is shown in Figure 1. It shows the structure of a small database concerning students, professors and courses. The diagram depicts a well known situation at a university, where professors teach courses that are taken by students. Both professors and students are persons with a name, who live at an address and are born on a certain date. No two persons at the university have the same name and date of birth. Students finance their education by means of either a scholarship or a loan.

The diagram is composed of rectangles and arrows of various kinds. A rectangle represents an object type. In any instance of the database it stands for a set of objects of that type. Sets of objects corresponding to different object types are disjoint. The arrows represent property types. A property type stands, in an instance of the database, for a (mono- or set-valued) function between the set of objects at the base of the property arrow and the set of objects at its tip.

The graphical conventions used in Figure 1 are given in Figure 2 and will be explained below. In addition to object types and their functional relations also a number of (common) constraints can be expressed graphically. 

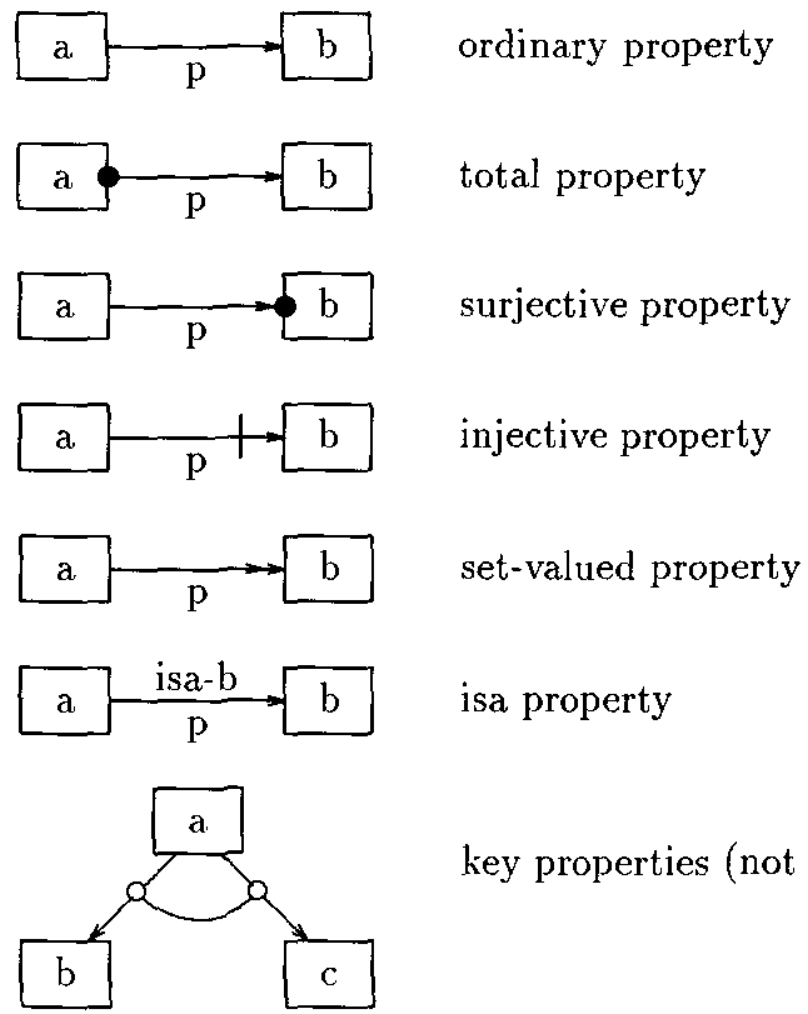

key properties (not necessarily total)

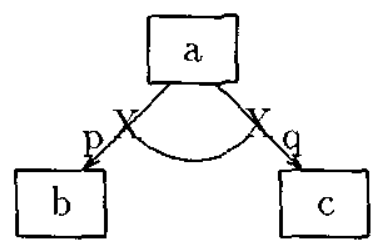

properties with mutually exclusive domains

Figure 2: Graphical Representation of Objects and Properties.

The information represented in Figure 1 may also be represented textually using a structure scheme. We will give the definition of this scheme first and then work it out for the University example.

\section{Definition 2.1 Structure Scheme}

A structure scheme is a 4 -tuple $\langle O, P, C, W\rangle$ where

$O$ : a finite set of names of object types.

$P$ : a triple specifying a finite set of property types; $P=\langle F, D, R\rangle$, where

$F$ is a finite set of names of property types: $O \cap F=\emptyset$.

$D$ is a function which maps a property type to the object type which is called the domain type of the property type; so $D \in F \rightarrow O$.

$R$ is a function which maps a property type to the object type which is called the range type of the property type; so $R \in F \rightarrow O$.

When a property is set-valued we say that the range is $O$, not $\mathcal{P}(O)$.

$C=\langle Q, U, X\rangle$, a triple specifying standard constraints : 
$Q:$ a function which assigns to every property type a number of attributes:

$Q \in F \rightarrow V_{\text {is_a }} \cup$

$\prod(\{<$ total,$\{T, \perp\}>,<$ injective, $\{T, \perp\}>,<$ surjective, $\{T, \perp\}>$,

$<$ set-valued, $\{T, \perp\}>\})$. $^{1}$

For set-valued properties we say that being total means that the property must not only be defined for every object of the domain type, but also that the corresponding set of objects in the range type is non-empty. For the injectivity and surjectivity we look at the objects in the range sets, not the range sets themselves. Injectivity then means that the property defines a partition on the range object type: $\forall a, b \in \operatorname{dom}(f): a \neq b \rightarrow f(a) \cap f(b)=\emptyset$. An injective setvalued property $f$ then can be thought of as the inverse of an ordinary property $f^{\prime}$ from $R(f)$ to $D(f)$. Surjectivity means that $R(f)=\bigcup\{f(a) \mid a \in \operatorname{dom}(f)\}$.

$U$ : a function which assigns to an object type $o \in O$ the subsets $k$ of $D^{-1}(o)$ of names of property types, called the keys of this object type, that together form an injective function from o to $k$, so $U \in O \rightarrow \mathcal{P}(\mathcal{P}(F))$. We do include singletons-sets containing a single, injective property type-in $U$, although they are already represented by $Q$. However, in our examples we will not mention the singletons explicitly. The keys in $U$ are minimal, of course.

$X$ : a function which assigns to an object type $o \in O$ the subsets of $D^{-1}(o)$ of names of property types, that have mutually exclusive domains, so $X \in O \rightarrow \mathcal{P}(\mathcal{P}(F))$.

$W$ : a set valued function with $\operatorname{dom}(W)=O$, called the object world function. If for $f \in F$ it holds that $Q(f) \in V_{\text {is_a }}$ then $W(D(f)) \subseteq W(R(f))$

The components $O$ and $P$ together specify a labeled, directed graph that gives the structure of the database in terms of object and property types. Component $C$ specifies a number of general constraints these types have to satisfy. $W$ is a function which associates a set of real world objects with (the name of) an object type. These sets do not have to be disjoint. On the contrary, if two object types are related to one another by an is_a relationship, the set of real world objects corresponding to the subtype is required to be a subset of the set of objects for the supertype. A single real world object thus may be represented in the database model by two or more objects that are related by is_a-relationships and represent the various roles the real world object plays in the model. $W$ allows one to make statements about the relation between sets of objects, but can, since it refers to real-world objects, not be formalized completely.

The $O$ and $P$ components of the structure scheme specify a labeled, directed graph, in which the object types are nodes and the property types correspond to labeled, directed edges. Not every graph specified this way is acceptable as a structure scheme. When we select the subgraph $\langle N, E\rangle$ based on the property types with an is_a-label with edges $E=\left\{f \in F \mid Q(f) \in V_{i s_{-} a}\right\}$ and nodes $N=\{o \in O \mid \exists\{f \in E \mid o=D(f) \vee o=R(f)\}\}$, this subgraph has to be free from cycles. Also, in $\langle N, E\rangle$, if an object has 2 or more supertypes (i.e. has 2 or more

\footnotetext{
${ }^{1}$ The symbol $\Pi$ denotes the Generalized Product: Let $P$ be a set valued function, then $\Pi(P)=\{p \mid p$ is a function with domain $\operatorname{dom}(P)$ and $\forall x \in \operatorname{dom}(P): p(x) \in P(x)\}$, also, $T=$ true, $\perp=$ false; $V_{\text {is_a }}$ is a set of (is_a-) labels, disjoint from $F$ and $O$.
} 
outgoing is_a edges) then these must have a common supertype. Furthermore, if there is no directed path from an object type $o_{1} \in N$ to an object type $o_{2} \in N$ then $o_{1}$ and $o_{2}$ correspond to disjoint sets of real-world objects: $W\left(o_{1}\right) \cap W\left(o_{2}\right)=\emptyset$.

The is_a properties are required to be total, injective and not set-valued. Every subtype object thus is mapped to precisely one, unique supertype object. Is_aproperties are given an is_a-label $l\left(l \in V_{i s_{-} a}\right)$ in addition to a name. When two is_a-properties have a common supertype as range type and have the same is_alabel, their range sets are disjoint. The range sets of is_a-properties with a common supertype, but with different is_a-labels are allowed to overlap.

To illustrate the definition of a functional database scheme we give the scheme version of the structure of (part of) a university database.

Example 2.1 University database (structure scheme) The components $\langle O, P, C, W\rangle$ for the example of Figure 1 are:

$O=\{$ address, name, date, person, professor, student, amount, course $\}$

$P=\langle F, D, R\rangle$, where

$F=\{$ lives at, called, born on, p, s, loan, scholarship, teaches, takes $\}$

$D=\{$ (lives at, person $),($ called, person $),($ born on, person $),(\mathrm{p}$, professor $)$, (s, student), (loan, student), (scholarship, student), (teaches, professor), (takes, student)\}

$R=\{($ lives at, address $),($ called, name $),($ born on, date $),(\mathrm{p}$, person $),(\mathrm{s}$, person $)$, (loan, amount), (scholarship, amount), (teaches, course), (takes, course) $\}$

$C=\langle Q, U, X\rangle$, where $\begin{aligned} & Q=\{(\text { lives at },(<\text { total }, \top>,<\text { injective }, \perp>,<\text { surjective }, \perp>,<\text { set-valued }, \perp> \\ & \text { ) },\end{aligned}$

(called, ( $<$ total, $T\rangle,<$ injective, $\perp>,<$ surjective, $\perp>,<$ set-valued, $\perp\rangle)$ ), (born on, $(<$ total, $T\rangle,<$ injective, $\perp\rangle,<$ surjective, $\perp\rangle,\langle$ set-valued, $\perp\rangle$ ) ),

$\left(\mathrm{p}\right.$, isa $\left._{\text {prof }}\right),\left(\mathrm{s}\right.$, isa $\left._{\text {stud }}\right)$,

(teaches, $(\langle$ total, $\perp\rangle,\langle$ injective, $\perp\rangle,\langle$ surjective, $\perp\rangle,\langle$ set-valued, $T\rangle$ ) ),

(takes, $(<$ total, $T\rangle,<$ injective, $\perp>,<$ surjective, $\perp>,<$ set-valued, $T\rangle$ )), (loan, $(<$ total,$\perp\rangle,<$ injective, $\perp\rangle,<$ surjective, $\perp\rangle,\langle$ set-valued, $\perp\rangle$ )),

(scholarship, $(<$ total, $\perp\rangle,<$ injective, $\perp\rangle,\langle$ surjective, $\perp\rangle$,

$<$ set-valued, $\perp>)$ ),

$U=\{($ person, $\{$ called, born on $\})\}$

$X=\{($ student,$\{$ loan, scholarship $\})\}$

$W=$ the function linking every object in the database to the corresponding object in the real world. This basically is the "meaning" of the database.

We require the takes property to be total, meaning that the set of courses taken by a student must be known and non-empty. We do not require the teaches property to be total, mostly to show the difference. 
From $U$ we see (as shown in Figure 1) that the key properties for a person are his (her) name (property called) and date of birth (property born on).

$X$ tells us that a student cannot have both a loan and a scholarship at the same time. In some sense, having such properties which exclude each other is an alternative to creating subtypes (using is_a properties) in some simple cases. We could have created subtypes student-with-loan and student-with-scholarship, both with the same is_a label, and that would have meant that a student cannot have both a loan and a scholarship. A person however can be both a professor and a student at the same time, because the is_a labels isa prof $_{\text {and }}$ isa stud $_{\text {are }}$ different.

The figure is fairly easy to understand, whereas a typical non-expert would not even begin to read the formal mathematical definition. The two are completely equivalent however. We use the figure to clarify the database design to the customer, while the mathematical definition is used in the transformation algorithm. In addition to the mathematical definition corresponding to the figure one can define a formal language for expressing constraints and queries. This has been done in [5] for instance. The mathematical definition is what is typically stored in the data dictionary.

Note also that the structure scheme we have given for the university database does not describe all the details that will be present in an actual implementation. We have omitted properties which are unimportant for describing the overall structure of the database, such as the representation of a course by means of a number and/or title. (Such details are needed later on, regardless whether the implementation is based on a relational or a functional DBMS.)

In order to transform a structure scheme into a relational scheme we first eliminate the set-valued functions.

Definition 2.2 Purely Functional Structure Scheme

A structure scheme is called purely functional if there are no set-valued properties (i. e. for all properties the set-valued attribute is $\perp$ ).

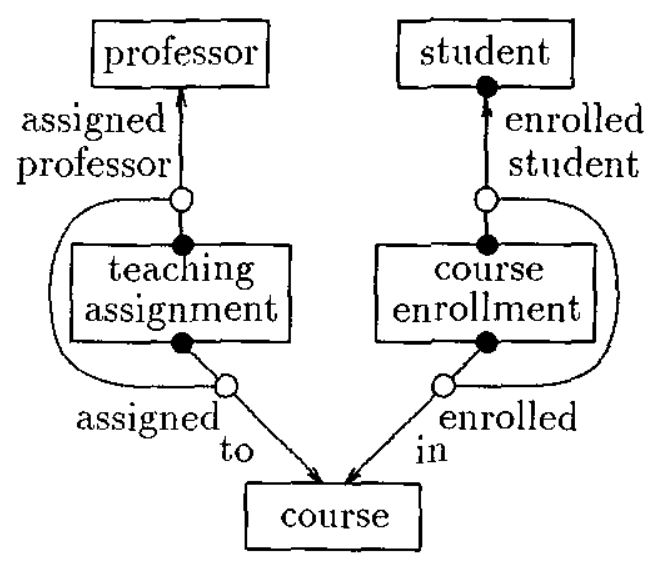

Figure 3: Purely Functional (part of the) University Database

A structure scheme can always be transformed into a purely functional scheme by replacing set-valued property types by an extra object type and two mono-valued 
total key-properties. Figure 3 shows how this is done in the university example. The figure shows that although we use a (double) arrow to draw a set-valued function the translation becomes symmetric: a professor may teach a number of courses, and a course may also be taught by a number of professors.

\section{Relational Representation Schemes}

Before we can present our transformation algorithm we need the definition of the target (or output) of that algorithm: a relational database scheme, which we call relational representation scheme because it is a representation of the structure scheme in an alternative but at least as powerful formalism. We first present the definition of a relational representation scheme and then discuss its components.

\section{Definition 3.1 Relational Representation Schema}

A relational representation scheme for a purely functional structure scheme $<O, P, C, W>$ is a 6 -tuple $\langle E, A, V, I, H, T>$ where:

$A$ : a finite set of names of attributes

$V:$ a function with $\operatorname{dom}(V)=A$, which maps every attribute name $a \in A$ to a set of values $V(a)$, called attribute range, sometimes also called the domain of the attribute.

$H$ : a (partial) function ${ }^{2}$, which maps object types $o \in O$ to a corresponding set of attribute names $H(o) \subseteq A$, so $H \in O \not \mathcal{P}(A)$.

$I$ : a function, which maps each object type $o \in \operatorname{dom}(H)$ to a corresponding set of attribute names $I(o)$, which is called the primary key of $o$, so $I \in \operatorname{dom}(H) \rightarrow$ $\mathcal{P}(A)$ and $\forall o \in \operatorname{dom}(H): I(o) \subseteq H(o)$.

$E$ : a (partial) function, which maps property types $f \in F$ to an injective function of attribute names to attribute names, such that:

$$
\begin{aligned}
& \forall f \in \operatorname{dom}(E): E(f) \in I(R(f)) \rightarrow H(D(f)) \text { and } \\
& \forall f \in \operatorname{dom}(E): \forall a \in I(R(f)): V(a)=V(E(f)(a)) .
\end{aligned}
$$

$T$ : a function which maps each object type to a function which maps the identifying (primary key-) part of the representation of a real world object to the object itself, so $\operatorname{dom}(T)=O$, and $\forall o \in O: T(o) \in \Pi(V \mid I(o)) \rightarrow W(o)$.

This definition shows some important differences between the functional database model and the relational model. The functional model at the level of the structure scheme is object-oriented, while the relational model is value-oriented. $V$ defines the domain of each attribute: a set of values. In the functional model we only have objects, although some objects do not correspond to physical real-world objects but rather to abstract objects (like a date for instance). The functional

\footnotetext{
${ }^{2}$ The symbol $\not$ is used to denote a partial function.
} 
model does not say how to print or represent a date, whereas the relational model does indicate the set of possible values.

In the functional model an object type o may have several keys- $U(o)$ may have more than one element-whereas we need one primary key in the relational representation scheme, to act as an object identifier and to simulate the functional relationships between objects.

$H$ represents the heading of the relation (or table) that corresponds to an object type. $H$ is a partial function because we only want to create a relation for object types with properties. A table which only contains dates for instance usually is of little use and a relation for it can be omitted provided that does not cause any loss of information.

To represent a property of an object we will include a representation of the range object of that property in the tuple for the object. The heading of the relation for an object type thus will include a set of attributes for representing each of the property types of which that object type is the domain type. When the range type of a property of an object type is itself an object type with properties of its own (hence it will be represented by a table) then we need a link between the attribute(s) corresponding to that property and the attribute(s) that identify the objects in the range type of that property. This link is provided by the renaming function $E$. Although the renaming is usually the identity this need not always be the case. In the purely functional university example we can use enrolled in as the attribute to indicate the course to which a course enrollment refers, whereas this attribute corresponds to some attribute of course, which we shall name courseno below. $E$ indicates that courseno is renamed to enrolled in in the course enrollment relation.

Finally, $T$ ensures that tuples in our relations correspond to objects in the real world, by mapping the primary key for an object of type $o$ to an object in $W(o)$, the real-world object it represents.

Before turning to the algorithm we show a possible relational representation scheme for the university database. This representation scheme correponds to the structure diagram of Figure 4, the purely functional version of the diagram of Figure 1 which has been made more complete by adding a few more details, such as the title of a course, the name of the street, and the number of the house in the address. (The reader is invited to verify that this relational representation is indeed the output of the algorithm.) Some elements in this relational scheme may not represent the typical use of the university database. It is always possible to change some attributes or relations, for instance to select more appropriate primary keys.

Example 3.1 Relational University database

The components $\langle E, A, V, I, H, T\rangle$ of a possible relational representation scheme are :

$A=\{\mathrm{nr}$, street, state, zip, city, name, birthdate, SS\#, empno, studno, loan, courseno, coursename, assigned professor, assigned to, enrolled student, scholarship, enrolled in\}

These are the names of the attributes we will use in our relations. Some of these attributes are not represented in the structure diagram of Figure 1, but shown in Figure 4. Also, there is no need to use the same names for attributes as for their "corresponding" properties in the structure scheme. In particular we have chosen to 
use birthdate instead of born on and name-the name of an object type- instead of called.

$$
\begin{aligned}
& V=\{(\mathrm{nr}, \text { numbers }),(\text { street, strings), (state, char [2]), (zip, numbers), (city, strings), } \\
& \text { (name, strings), (birthdate, dates), (SS\#, numbers), (empno, numbers), } \\
& \text { (studno, numbers), (loan, money), (scholarship, money), (courseno, numbers), } \\
& \text { (coursename, strings), (assigned professor, numbers), (assigned to, numbers), } \\
& \text { (enrolled student, numbers), (enrolled in, numbers)\} }
\end{aligned}
$$

where strings, char[2], numbers, dates, money denote the sets of all possible strings, strings of 2 characters, numbers, dates, and amounts of money. Again, the name of the domain of an attribute need not be the same as the name we used for the object type in the structure scheme. For instance, dates refers to the set of values we use to represent objects of type date, and values of type money represent an amount.

$$
\begin{aligned}
H= & \{(\text { person },\{\mathrm{nr}, \text { street, state, zip, city, } \mathrm{SS} \#, \text { name }, \text { birthdate }\}), \\
& \text { (student, }\{\text { studno, } \mathrm{SS} \#, \text { loan, scholarship }\}),(\text { course },\{\text { courseno, coursename }\}), \\
& (\text { teaching assignment, }\{\text { assigned professor, assigned to }\}), \\
& (\text { professor, }\{\text { empno, SS\#\}),(course enrollment, }\{\text { enrolled student, enrolled in }\})\}
\end{aligned}
$$

$H$ defines headings of the tables. We have chosen not to define relations for the object types name, date and amount since they have no properties. There is also no table for address because there is only one property which refers to address and there is no (nontrivial) key for address. In other words: all information about adresses can be represented in the person relation. Note also that we have added attributes (empno, studno, SS\#, courseno and coursename) which are not present in the structure scheme. It is common to omit properties in a structure scheme to trim down the graphical representation.

$$
\begin{aligned}
I= & \{(\text { person },\{\text { SS\# }\}),(\text { professor },\{\text { SS\# }\}),(\text { student },\{\text { SS\# }\}),(\text { course },\{\text { courseno }\}), \\
& (\text { teaching assignment, }\{\text { assigned professor, assigned to }\}), \\
& (\text { course enrollment, }\{\text { enrolled student, enrolled in }\})\}
\end{aligned}
$$

$I$ defines the primary keys. We have chosen the social security number to be the primary key of the person relation. Because professor and student are specializations of person (they are connected to person through an is_a relationship) they inherit the same primary key. In an implementation however one may wish to use the empno as primary key for professor and the studno for student. This means that a teaching assignment could consist of an empno and a courseno, or in other words that empno could be used as a foreign key in the teaching assignment relation.

Note that the objects in the structure scheme may have more than one key. Our relational representation scheme does not have an equivalent to $U$, the set of all keys for the objects, as present in the structure scheme. The constraints specified at the structure scheme level of course still hold at the representational level and do not have to be repeated there. In general one will describe the constraints in a formal language, like in [5]. These constraints are not essential for our transformation algorithm, since they do not affect the structure of the model.

$$
\begin{aligned}
E= & \{(p, \operatorname{id}(\{\operatorname{SS\# }\}),(\mathrm{s}, \mathrm{id}(\{\mathrm{SS} \#\}),(\text { assigned professor },\{(\text { SS\#, assigned professor })\}), \\
& (\text { assigned to },\{(\text { courseno, assigned to })\}),(\text { enrolled in },\{(\text { courseno, enrolled in })\}), \\
& (\text { enrolled student, }\{(\text { SS\#, enrolled student })\})\}
\end{aligned}
$$


We see that in our example a renaming function has been defined only for property types relating object types for which a relation has been constructed. This usage of renaming is a reflection of the referential integrity constraints that functions between sets of objects have to satisfy. The renaming function for a property type $p$ maps the attributes in the primary key of $R(p)$ to the corresponding attributes in $D(p)$. For the is_a-properties in our example this mapping turns out to be trivial. For a property type such as enrolled in it is a little less trivial. Renaming becomes necessary when one object type is related to another one by more than one property type. Consider for example an object type period which is related by property types begin and end to an object type date. The relation for period then may contain attributes such as begin_of_period and end_of_period which are renamed from the primary key attribute, say date, of the relation for the object type date. Attributes that are mapped to one another by a renaming function have the same attribute range.

$T=$ a complicated function which maps each object type to a function from the set of possible primary-key values for the representation of an object to the set of real-world objects. Basically, this is the equivalent of the object world function, but now for the relational representation. If the "person" object with name "John Doe", and born on 1/1/1950 refers to a real person, then the tuple in the person-table with name-value "John Doe" and date of birth "1/1/1950" must refer to the same real person.

\section{The Transformation Algorithm}

The transformation from a functional structure scheme to a relational representation scheme can only be completely automated if the structure scheme is complete. Often one omits a number of properties to keep the graphic representation small. Figure 4 shows a complete, purely functional structure scheme for the university database. We have chosen object names almost at random for (what will become) the attributes.

We now turn to the exact description of the algorithm which takes as input a functional database scheme, and produces an equivalent relational scheme in BoyceCodd Normal Form (and, because of the absence of multivalued dependencies and join dependencies, also in Fifth Normal Form, see also [9]). We will first present a summary of the algorithm. In the sections that follow the most important steps in the algorithm are discussed in more detail.

\subsection{Summary of the Algorithm}

To summarize the algorithm we enumerate the basic steps and we apply them to the university example.

1. Create a purely functional scheme, equivalent to the given structure scheme. For the university example, see Figure 4.

2. Create a relation name $o$ for each object type $o$. 


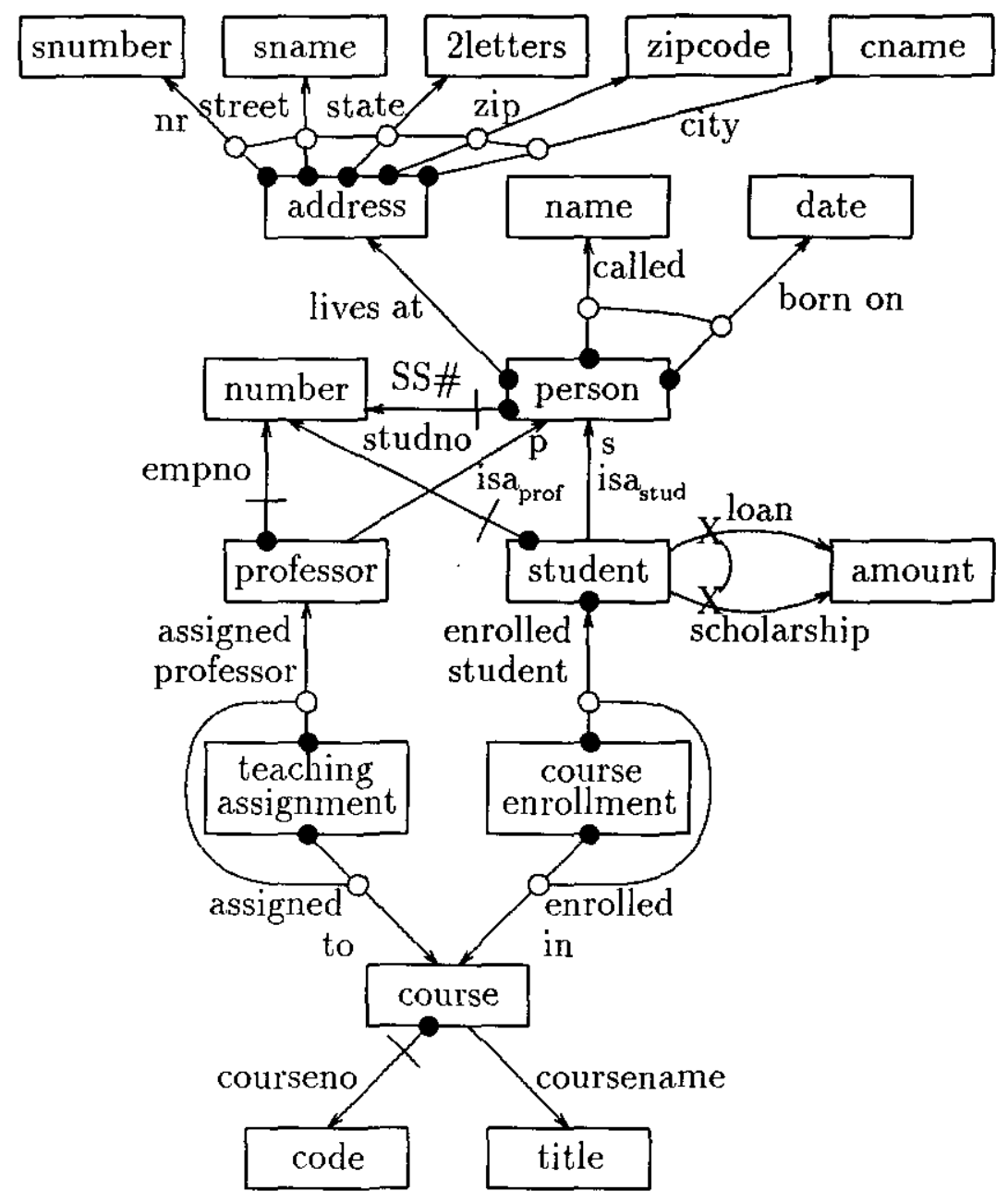

Figure 4: Complete, Purely Functional University Structure Diagram

3. Determine the level of each object type. The level determines the order in which primary keys can be assigned.

4. Every relation representing an object type of level 0 gets one attribute which we call $o$, and which is the primary key of that relation.

5. Determine the primary key of each relation, starting at level 1, and working in ascending order. The primary key is determined as follows :

(a) For an object type with an is_a property we take the primary key of the superclass.

(b) For an object type with a total key (but without an is_a property) we take the union of the primary keys of the relations corresponding to the object types that are the range of the key-properties.

(c) For the remaining object types we create a single new attribute that will become the primary key.

6. Determine the other attributes of each relation, again starting at level 0 , and working in ascending order. The attributes are created much in the same way as we did for the primary key. 
7. Remove the relations corresponding to object types without properties, when this can be done without loss of information. A relation can be removed when the range sets of property types that have the corresponding object type as range type, are required to either separately or jointly cover the set of range objects in any state of the database.

Note that since we only have functional relationships between object types and that every (non-attribute) object type is represented by a separate relation there are no functional dependencies within a relation that are not key-dependencies. Hence the relational database schemes we generate are always in Fifth Normal Form.

\subsection{Removing Set-Valued Properties}

Because of the widely accepted (and enforced) First Normal Form all values that can occur in relation instances must be atomic. Structured (i. e. tuple-valued) and/or set-valued attributes are prohibited. In the transformation of a functional database scheme to a relational scheme we wish to represent functional relationships by (one or more) attributes. To avoid set-valued attributes we must eliminate set-valued functions from the structure scheme first. This is done by transforming the functional scheme into a purely functional scheme (see Definition 2.2) by replacing every set-valued function by a combination of an extra object type and two mono-valued total key-properties. The result of applying this transformation to the set-valued functions teaches and takes in Figure 1 is shown in Figure 3.

Let $o_{1}, o_{2} \in O$ and let $f \in F$ be a property name such that $D(f)=o_{1}$, $R(f)=o_{2}$ and $Q(f)$ (set-valued) $=\mathrm{T}$. Suppose first that $f$ is not injective. The replacement of the set-valued function $f$ then works as follows:

- Remove all information concerning $f$ from the components $P=\langle F, D, R\rangle$ and $C=\langle Q, U, X\rangle$ of the structure scheme.

- Add a new object $o$ to $O$. For example, an object type teaching assignment will be added as part of replacing the set-valued property teaches.

- Add two new property names $f_{1}$ and $f_{2}$ to $F$. In our example we introduced the property names assigned professor and assigned to.

- Add $\left(f_{1}, o\right)$ and $\left(f_{2}, o\right)$ to $D$.

- Add $\left(f_{1}, o_{1}\right)$ and $\left(f_{2}, o_{2}\right)$ to $R$.

- Add $\left(f_{1},(<\right.$ total, $T\rangle,<$ injective, $\perp>,<$ surjective, $\alpha>,<$ set-valued, $\left.\left.\perp>\right)\right)$ to $Q$ where $\alpha=\mathrm{T}$ if $f$ is total and $\perp$ if it is not. According to Definition 2.1 being total means that the sets in the range must all be nonempty. In the university example the teaches property would only be total if every professor would be linked to a nonempty set of courses. In that case every professor would be the assigned professor for at least one teaching assignment.

- Add $\left(f_{2},(<\right.$ total, $\top\rangle,<$ injective, $\left.\perp\right\rangle,<$ surjective, $\left.\alpha\right\rangle,<$ set-valued, $\left.\left.\left.\perp\right\rangle\right)\right)$ to $Q$, where $\alpha=T$ if $f$ is surjective and $\perp$ if it is not. If every course occurs in the set of courses taught by some professor, then every course will be assigned to some professor in some teaching assignment. 
- Add $\left(o,\left\{f_{1}, f_{2}\right\}\right)$ to $U$. So for every course a professor teaches a unique teaching assignment has to be introduced.

By applying the composition of the inverse of assigned professor and assigned to to an object in the range of assigned professor (= dom(teaches)) we obtain the set of course-objects taught by the corresponding professor. The set-valued property teaches thus is effectively replaced by the equivalent set-valued composition $f \circ g^{-1}$ with $f=$ assigned to and $g=$ assigned professor.

From the example above it can be seen that the procedure may be simplified when $f$ is injective. The injectivity constraint would be carried over to $f_{2}$ implying that every object in o corresponds to one, unique object in $o_{2}$. Property $f_{2}$ now does not contain any new information. It is therefore simpler to replace the set-valued property $f$ by an ordinary property $f^{\prime}$ going in the opposite direction. So:

- Remove all information concerning $f$ from the components $P=\langle F, D, R\rangle$ and $C=\langle Q, U, X\rangle$ of the structure scheme.

- Add one new property type name $f^{\prime}$ to $F$.

- $\operatorname{Add}\left(f^{\prime}, o_{2}\right)$ to $D$.

- Add $\left(f^{\prime}, o_{1}\right)$ to $R$.

- Add $\left(f^{\prime},(<\right.$ total, $\alpha>,<$ injective, $\perp>,<$ surjective, $\beta>,<$ set-valued, $\left.\perp>)\right)$ to $Q$ where $\alpha=T$ if $f$ is surjective and $\perp$ if it is not, and $\beta=T$ if $f$ is total and $\perp$ if it is not.

As an example consider the case where teaches in Figure 1 is required to be injective. This means that none of the courses taught by one professor are taught by any of the other professors. Teaches is then replaced by an ordinary property from course to professor with a suitable name such as taught_by.

The above procedure is repeated until all set-valued properties have been eliminated.

Some information (from $U$ and $X$ ) is not accounted for during the elimination of set-valued properties. Using a formal constraint-language (see [5]) one can still describe the altered constraints, but we can no longer represent them using our graphical representation.

\subsection{Selection of Relations}

Perhaps the most crucial part in the transformation is the selection of the relations that are going to represent the object types of the structure scheme.

In order to automate this step it is important to have a complete structure scheme, i.e. a scheme in which all properties we wish to convert to attributes in the relational scheme are present. Whether one just keeps these properties in mind or actually draws them (like we did in Figure 4) is irrelevant. Also, in a more interactive version of this algorithm one may relax this constraint, as we in fact only need to know whether an object has properties, not which or how many of them. 
In general all object types which are the domain of a property (i.e. which have outgoing arrows in the graphical representation) are candidates for being represented by a relation. However, some of these candidates may be uninteresting, and before actually implementing the relational scheme one must examine the scheme and eliminate the relations one considers useless. For instance, if the only key of an object type is the set of all its property types, then the only way to identify an object of that type is by giving (i. e. repeating) all its properties. In the university example the person object type has a lives at property which refers to an address object type. The only way to identify an address in our example is by giving all its properties. Hence in a relation instance all information about people's addresses would be contained in the person relation and also in the address relation. Furthermore, none of the properties of address have a range type that has properties of its own. Since there is no use for such a redundant address relation we do not generate it.

For the remainder of the algorithm we assume that we have a relation $o$ for every object type $o \in O$. As a final step of the algorithm the relations corresponding to object types without properties are thrown away. So from $H, E$ and $I$ (defined below) we delete the elements that correspond to those relations.

\subsection{Choosing Primary Keys}

The most important problem in transforming functional schemes into relational representations is the identification of objects (and the translation of these id's into primary keys). In an object-oriented database (of which functional databases are a special kind) every object has an object-identity, which uniquely identifies it. In a relational database there are no "objects", only values (grouped into tuples). The aim of our transformation algorithm is to find a way to represent objects by means of tuples. This means that somehow the notion of object-identity has to be simulated using values. We do this by introducing primary keys. Depending on how the object-identity can be deduced from the structure scheme we will use different representations of the identity using primary keys.

The algorithm will be such that when an object type is a subtype of another object type, it will inherit the primary key of its supertype. When an object type is not a subtype, but satisfies a total key constraint, we choose to construct the primary key of this type on the basis of the primary keys of the range types of its key properties. If neither of the previous two cases is applicable the identity of the objects is considered to be internal to the object type, i.e. it can not be derived from that of other object types and an (artificial) one-attribute primary key is introduced. Note that we could apply this last alternative to every object type. This however we consider contrary to the semantics of the subtyping relations between object types and - in most cases - of the total key constraints.

To deternine the order in which we assign primary keys to the relations representing object types we assign levels to the object types. In this way we in effect determine what is known as the "object identity graph" [10]. The assignment algorithm we use is an adapted version of the topological sort algorithm. The levels make it possible to introduce primary keys in a single pass through the structure diagram. In what follows we only need that part of the structure diagram consisting of the object types, the properties with an is_a-label and those total properties 
that are part of a key, provided this key contains only total properties. The object types without such properties are of level 0 . The remaining object types fall into two categories: those with an is_a-property and those without. An object type with an is_a-property is assigned the level of its supertype increased by one. When an object type is directly related to more than one supertype it gets the largest of the supertype levels increased by one. Because of the restrictions we imposed following Definition 2.1 on specialization relations this procedure leads to an unambiguous level for every subtype.

The levels of the object types without is_a-properties but with a total key are in essence determined as follows. The object types with properties of which the range are object types of level 0 are of level 1 . Those with properties of which the range are of level at most 1 are of level 2 . We repeat this procedure until we have assigned a level to all object types of the structure scheme.

In case an object type is required to satisfy more than one total key constraint we take that key for which the levels of the associated range types are known. When there is more than one such key we choose arbitrarily among the keys with the smallest number of properties. Since we did not restrict the assignment of total key constraints in any way other than having to be minimal, we may run into a mutual dependency among several object types with a total key constraint.

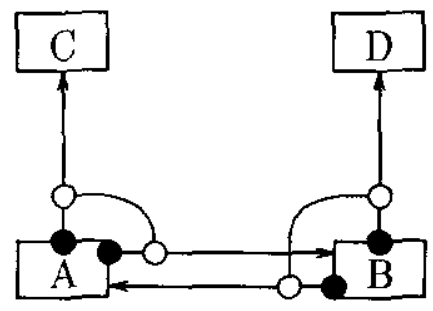

Figure 5: Mutually Dependent Keys

Figure 5, for example, shows a situation in which the level of object type A can not be determined before the level of $B$ is known, which can only be determined after the level of $A$ has been established. In such case we break the cycle by choosing arbitrarily among the object types involved with the largest number of key properties and assign it level 0 .

Then we assign primary keys to the object types, starting with the objects of level 0 (in any order), then continuing with the object types of level 1 etc. $^{3}$

For each object type there are three possible ways they can be identified. We can only assign one primary key to every relation, so we try each of the following possibilities in the order in which they are given below:

1. If there is an is a property with name $f$ from $o$ to $o^{\prime}$ then $I(o)=I\left(o^{\prime}\right)$. Note that $o^{\prime}$ is of a lower level than $o$ so $I\left(o^{\prime}\right)$ has been determined before we try to define $I(o)$.

Note also that if there is another is_a property from $o$ to an object type $o^{\prime \prime}$ then (see the remarks after Definition 2.1) $o^{\prime}$ and $o^{\prime \prime}$ must have a common supertype

\footnotetext{
${ }^{3}$ The assignment of levels to object types is the reason why we need a complete structure scheme. In an interactive implementation of the algorithm one could ask for the level of object types that seem to be of level 0 . The user could then tell the system that an object type is of a higher level because of properties that were omitted in the structure scheme.
} 
and inherit the same primary key (from the common supertype) regardless the is_a property we choose to use for determining $I(o)$.

2. If $o$ has one or more total keys, i. e. $\exists k \in U(o): \forall f \in k: Q(f)($ total $)=T$ and one of these was chosen to assign a level to $o$, we generate a primary key for the relation $o$ from that key. Let $k \in U(o)$ be the chosen key, then $I(o)=\bigcup_{f \in k}\left\{f-o^{\prime} \mid o^{\prime} \in I(R(f))\right\}$. So we copy the names of the attributes in the primary keys of the relations corresponding to the range of the properties in $k$, and add the name of the property to the names of these attributes, to keep names unique. We also add $\bigcup_{f \in k}\left\{\left(o^{\prime}, f-o^{\prime}\right) \mid o^{\prime} \in I(R(f))\right\}$ to $E$ to provide the link between the names of attributes in $R(f)$ and in $o$ needed for keeping track of referential integrity constraints.

3. If $o$ has no total $\mathrm{key}^{4}$ or was assigned level 0 , then there is no representation of the notion of object-identity of $o$ by means of values. We then create a new artificial attribute, which we call $o$, with a sufficiently large domain (e.g. the natural numbers) and we let $I(o)=\{o\}$.

\subsection{Naming Attributes and Domains}

The object types of level 0 that don't have properties of their own, i.e. object types $o \in O \backslash \operatorname{mng}(D)$, are not represented by relations. They are atomic, and represented by values. For every such object type we use the name of the object for the domain, i. e. the set of possible values representing the objects of this type. Therefore it is advisable to use meaningful names for these object types. When implementing the relational scheme one must associate types known to the DBMS to the domains. Different object types can be implemented using the same domain type.

For the assignment of attributes to relations we proceed in the same order as for the assignment of primary keys. For each object $o$ we create attributes as follows:

For every property name $f$ such that $o=D(f)$ we add the same number of attributes to $H(o)$ as are used in the primary key of the representation of $R(f)$, and, like we did for the primary keys, we add the name of the property $f$ to the names of the attributes of the primary key of the relation representing $R(f)$. (If $R(f)$ is an object of level 0 then we need one attribute, if $R(f)$ is of a higher level then we need $|I(R(f))|$ attributes.) Again, we add elements to $E$, describing the link between the attributes in $R(f)$ and in $o$.

\subsection{Boyce-Codd Normal Form}

Functional Structure Schemes only contain one type of "classical" constraints: keydependencies. An object is identified by the values for its key attributes. Key dependencies are a special case of a more general constraint: the functional dependency $(\mathrm{fd})$.

\footnotetext{
${ }^{4}$ Even if $o$ has a key one may choose to generate an "artificial" primary key consisting of only one attribute, by adding a key-property to the structure scheme. This is most useful when the existing total keys all consist of a large number of properties.
} 
In relational database theory fd's have been studied extensively. The existence of some fd's can enable a database designer to identify redundancy, and by decomposing relations (vertically) one can eliminate this redundancy. Different Normal Forms have been defined which indicate that some kinds of redundancy cannot occur in databases that satisfy the normal form. The most popular normal form which only looks at fd's is the Boyce-Codd Normal Form (BCNF). A relational scheme satisfies the $\mathrm{BCNF}$ if all functional dependencies in the scheme are key-dependencies. Since our algorithm generates one relation for every object type, and the only fd's we can express in the functional model are key-dependencies, the constraints of the functional model roll over into key-dependencies in the relational representation scheme. Hence our representation schemes are in BCNF.

In literature $[2,3]$ other constraints are described, such as multivalued dependencies (mvd) and join-dependencies ( $\mathrm{jd}$ ), leading to other normal forms (4-th and 5 -th normal form), which are all equivalent to BCNF plus some requirements for the mvd's and/or jd's. Since the relational schemes that are generated by our algorithm do not contain mvd's and jd's these schemes automatically also satisfy 4-th and 5-th normal form.

\section{Conclusions and Future Research}

We have presented an algorithm for transforming functional database schemes into relational representation schemes. This algorithm can be (and has been) implemented to run without user intervention. The generated relational database schemes are in Fifth Normal Form.

The relational representation schemes can be (and usually will be) modified before actually implementing them. We have given an example of redundant relations (the address relation), which one may wish to preserve or eliminate. We have also shown how to alter the functional scheme in order to avoid the duplication of keys with a large number of attributes.

In [5] a formal constraint language has been presented which can be used in conjunction with our graphical representation of database schemes, in order to capture constraints for which we have no graphical counterpart.

This transformation algorithm has been used successfully for designing and implementing relational database schemes while using functional schemes to present the design to non-experts (e.g. the customers).

In the near future our research will focus on the transformation of schemes in more general semantic database models to relational schemes. The IFO Model [10] is a prime candidate. We will also study the transformation to the Nested Relational Model $[11,12]$ which is a likely successor for the Relational Model as far as commercial database systems for the near future are concerned.

\section{References}

[1] Codd E.F., A Relational Model of Data for Large Shared Data Banks. Communications of the ACM 13:6, pp. 377-387, June 1970. 
[2] Paredaens J., P. De Bra, M. Gyssens, D. Van Gucht, The Structure of the Relational Database Model. EATCS Monographs on Theoretical Computer Science 17, Springer Verlag, 1989.

[3] Ullman J.D., Principles of Database and Knowledge-Base Systems, Volume I. Computer Science Press, Rockville, MD, 1988.

[4] Shipman D., The Functional Data Model and the Data Language DAPLEX. ACM Transactions on Database Systems 6:1, pp. 140-173, 1981.

[5] Aerts A.T.M., P. De Bra, K. M. van Hee, Combining the Functional and the Relational Model, Proc. of the CSN-90 Conference, pp. 1-16, Utrecht, 1990.

[6] Aerts A.T.M., K. M. van Hee, Modelling with a Functional Database Model. Informatie 12.89, 1989 (in Dutch).

Aerts A. T. M., G. Alblas, K. M. van Hee, Conceptual Modelling of Information Systems. Academic Service, Schoonhoven, 1991 (in Dutch).

[7] Teorey T. J., D. Yang, J. P. Fry, A Logical Design Methodology for Relational Databases Using the Extended Entity-Relationship Model. Computing Surveys 18(2), pp. 197-222, 1986.

See also: Teorey T.J., Database Modeling and Design, Morgan Kaufmann Publishers, Inc., San Mateo, CA., 1990.

[s] Hull R., R. King, Semantic Database Modeling: Survey, Applications, and Research Issues, ACM Computing Surveys, Vol. 19, No. 3, 1987.

Peckham J., F. Maryanski, Semantic Data Models, ACM Computing Surveys, Vol. 20, No. 3, 1988.

[9] De Troyer O. R. Meersman, Transforming Conceptual Schema Semantics to Relational Data Applications. Information Modelling and Database Management. Ed. H. Kangassallo. Springer Verlag, 1987.

[10] Abiteboul S., R. Hull, IFO : A Formal Semantic Model, ACM Transactions on Database Systems, 12, pp. 525-565, 1987.

[11] Arisawa H., K. Moriya, T. Miura, Operations and the Properties on Non-FirstNormal-Form Relational Databases, Proc. of the 9th International Conference on Very Large Data Bases, Florence, pp. 197-204, 1983.

[12] Fisher P.C., S.T. Thomas, Operators for Non-First-Normal-Form Relations, Proc. of the IEEE Computer Software and Applications Conference, Chicago, pp. 464--475, 1983.

\section{A The University Example Revisited}

In this appendix we present a DAPLEX formulation of the University Database Example from Figure 1. The structure of the object system is captured by a number of (stored) entity types:

DECLARE Person ()$\longrightarrow$ ENTITY 
DECLARE Address() $\longrightarrow$ ENTITY

DECLARE Name ()$\longrightarrow$ ENTITY

DECLARE Date ()$\longrightarrow$ ENTITY

DECLARE Amount ()$\longrightarrow$ ENTITY

DECLARE Course ()$\longrightarrow$ ENTITY,

two subtype relations:

DECLARE Professor ()$\longrightarrow$ Person

DECLARE Student ()$\longrightarrow$ Person,

and a number of single-valued and multivalued functions:

DECLARE Lives_at(Person) $\longrightarrow$ Address

DECLARE Called(Person) $\longrightarrow$ Name

DECLARE Born_on(Person) $\longrightarrow$ Date

DECLARE Loan(Student) $\rightarrow$ Amount

DECLARE Scholarship(Student) $\longrightarrow$ Amount

DECLARE Teaches(Professor) $\longrightarrow$ Course

DECLARE Takes(Student) $\longrightarrow$ Course

The example of Figure 1 is at the level of objects. This feature is reflected in the DAPLEX-definition by the fact that no printable types such as STRING occur in the definition. We further note that the meaning of subtype in our version of the functional datamodel differs from the one used by Shipman. In our version a subtype-object is closely related (in fact synonymous) to, but distinct from the supertype-object it corresponds to and from which it inherits all the properties. These two objects however correspond to the same object in the real world (via the mapping W). In Shipman's conception this is modeled by declaring for example Student to be of type Person.

Every function has an inverse in the model; these are defined as derived functions:

DEFINE Lives_at_inv(Address) $\longrightarrow$ INVERSE OF Lives_at(Person)

DEFINE Called_inv $\longrightarrow$ INVERSE OF Called(Person)

DEFINE Born_on_inv $\longrightarrow$ INVERSE OF Born_on(Person)

DEFINE Loan_inv $\longrightarrow$ INVERSE OF Loan(Student)

DEFINE Scholarship_inv $\longrightarrow$ INVERSE OF Scholarship(Student)

DEFINE Taught_by $\longrightarrow$ INVERSE OF Teaches(Professor)

DEFINE Taken_by $\longrightarrow$ INVERSE OF Takes(Student)

In Figure 1 also a number of constraints have been specified; the totality constraints can be formulated as:

DEFINE CONSTRAINT Called_total() $\longrightarrow$ FOR AIL Person SOME Name

SUCH THAT Name $=$ Called (Person) EXISTS

DEFINE CONSTRAINT Born_on_total() $\longrightarrow$ FOR ALL Person SOME Date

SUCH THAT Date $=$ Born_on(Person) EXISTS

DEFINE CONSTRAINT Lives_at_total ()$\longrightarrow$ FOR ALL Person SOME Address

SUCH THAT Address $=$ Lives_at(Person) EXISTS

and

DEFINE CONSTRAINT Takes_total ()$\longrightarrow$ COUNT(Takes(Student)) $>0$ 
The formulation of the exlusiveness and uniqueness constraints requires a few intermediate steps in the form of derived functions:

DEFINE LoanStudent ()$\longrightarrow$ Student SUCH THAT FOR SOME Amount Loan $($ Student $)=$ Amount

DEFINE SshipStudent ()$\longrightarrow$ Student SUCH THAT FOR SOME Amount Scholarship (Student $)=$ Amount

DEFINE LoanShip ()$\longrightarrow$ INTERSECTION OF LoanStudent, SshipStudent

DEFINE CONSTRAINT ExclusiveFunds ()$\rightarrow$ COUNT(LoanShip) $=0$

DEFINE NameDate ()$\longrightarrow$ COMPOUND OF Name,Date

DEFINE NameDatePairs(Person) $\longrightarrow$ NameDate SUCH THAT

FOR SOME Person Born_on(Person $)=$ Date $($ NameDate $)$

AND Called (Person $)=$ Name $($ NameDate $)$

DEFINE CONSTRAINT UniqueNameDate() $\longrightarrow$

COUNT $($ Person $)=\operatorname{COUNT}($ NameDatePairs $)$

For the latter constraint definition to work the earlier totality constraints on Called and Born_on are needed. 